\title{
Biventricular Pacemaker
}

National Cancer Institute

\section{Source}

National Cancer Institute. Biventricular Pacemaker. NCI Thesaurus. Code C80460.

A implantable cardiac pacemaker with an additional third lead placed in the left ventricle that is designed to keep the contractions of the ventricles synchronized with each other. 